\title{
Vision based behavior verification system of humanoid robot for daily environment tasks
}

\author{
Kei Okada, Mitsuharu Kojima, Yuichi Sagawa, Toshiyuki Ichino, Kenji Sato and Masayuki Inaba \\ Graduate School of Information Science and Technology, The University of Tokyo \\ 7-3-1, Hongo, Bukyo-ku, Tokyo, Japan \\ Email: k-okada@jsk.t.u-tokyo.ac.jp
}

\begin{abstract}
This paper describes integrated/intelligent humanoid robot system for daily-life environment tasks. We have realized complex behaviors of a humanoid robot in dailylife environment based on motion planner technique using an environment and manipulation knowledge. However in order to adapt to unknown or dynamic situations, sensor based behavior variation is essentially important. In this paper, we present a design and implementation of sensor based behavior verification system using an environment and manipulation knowledge, which is also used in manipulation motion planner. We also present software architecture that allows us to write a single stream code to perform complex concurrent humanoid motions. By using this architecture, sensor based verification functions are easily integrated in motion generation functions. Finally, we demonstrated a water-pouring task and a dishwashing task of the life-sized humanoid robot HRP2-JSK in a real environment while verifying its own motion.
\end{abstract}

\section{INTRODUCTION}

The progress of intelligent humanoid software is considered as followings.

1) Step 1: intelligence that manages its own body

2) Step 2: intelligence that manipulates tools through its own body

3) Step 3: intelligence that handles target objects by manipulating tools through its own body

In order to advance from walking and dancing behavior research of a humanoid robot(Step 1) to object handling and tool manipulating behaviors(Step 2,3), a humanoid robot system is required to manage a representation of target tools and objects. Of course, there exist developmental learning approach without given models [1], it seems to take long time to develop it.

Our approaches is to consider the necessary information from humanoid daily-life environment task experiment in order to develop humanoid intelligence as following steps, (a) a developer gives all information needed, (b) a robot obtain necessary information (c) a robot thinks what kind of information is needed.

The task of intelligence that manages its own body is navigation. In this level, researches on (a)a robot navigation upon given maps [2] and (b)a robot build maps by itself [3], [4] or a robot examine a correctness of an obtained map [5] are carried out.

However, there is little research on humanoid intelligence, which handles and manipulates tools through its own body.
There exists tool-handling research using hand eye systems in early robotics researches on assembly tasks in block world, however there is few for current research using humanoid robot in daily-life environment tasks with tools and objects that we use in everyday life [6]-[8]. We have developed dailylife environment behavior of humanoid robot as sweeping, vacuuming, pouring water from kettle, carrying tray, using washing machine, dish washing and so on [9].

This paper describes vision based behavior verification system in order to realize humanoid robots that adapt to change in an environment. Sensory-based behavior verification provides robust motion execution without having perfect knowledge of an environment.

The overall of this paper presents behavior verification system based on manipulation motion planner system. 3D shape information and manipulation knowledge (Section II) are utilized to navigate sensory information to verify motions (Section III). Multilayered behavior management architecture provides simple programming model (Section IV). Finally, we demonstrated a water-pouring task and a dishwashing task with developed system (Section V).

\section{TOOL MANIPULATION MOTION PLANNER USING MANIPULATION KNOWLEDGE}

A motion planner enables high performance on humanoid robot behaviors [10], [11]. However the most of them deal with navigation tasks and a few tackled manipulation behaviors [12], although they are required in daily-life tasks frequently. The difficulty is to develop a representation of tool manipulation planner, since the way we grasp and manipulate an object is not as simple as navigation tasks.

This section describes a tool manipulation motion generation method and an object representation for tool manipulation behavior of a humanoid robot.

\section{A. Object and environment model with manipulation knowl- edge}

Tool manipulation behavior of humanoid robots can be modeled as "a robot standing at certain position controls whole body joint angles while holding the target object".

Therefore, manipulation knowledge required for generating tool manipulation behavior are followings.

- Spot information: A coordinate on the floor where a robot stands when manipulate a tool. 

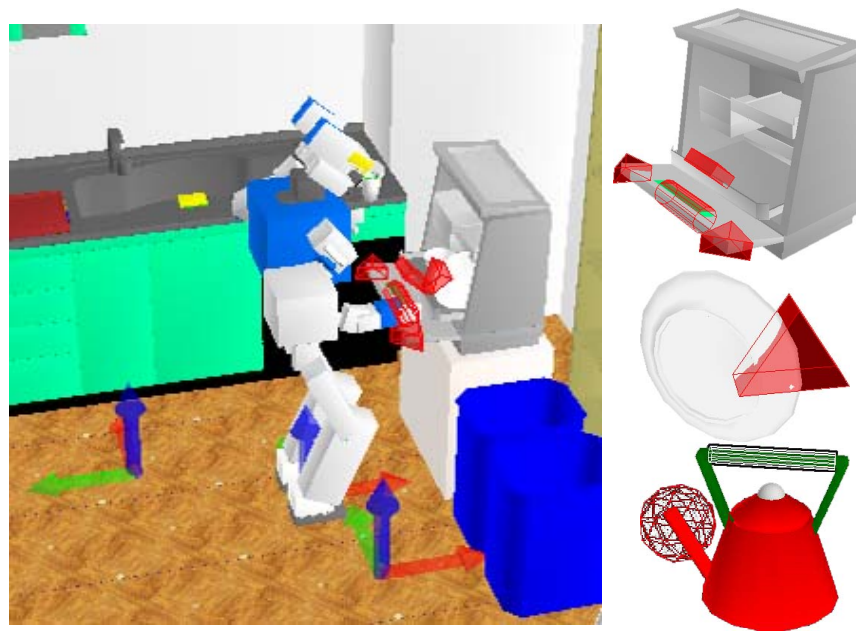

Fig. 1. An example of object and environment model with manipulation knowledge in kitchen task environment

Three arrows on the floor show spot information. Right figures show 3D shape model and handle information which is displayed as red triangles and cylinders. A red sphere in the kettle figure shows manipulation information.

- Handle information: Coordinates on an object and constraints which a robot reaches

- Manipulate information: A reference coordinates when a robot manipulates an object.

Constraints of Handle information are following five type. One is a 6 D.O.F. constraint with position and rotation. The other is a 3 D.O.F constraint with position. The rests are 5 D.O.F constraints with 3 axes in position and 2 axes in rotation that has rotational freedom along with $\mathrm{x}, \mathrm{y}, \mathrm{z}$ axis respectively. These constraints correspond to grasp, pinch, and pick behaviors of human.

Fig.1 shows an example of object and manipulation knowledge in kitchen task environment. Three arrows on the floor show locations of spot information These spots represent standing locations when a robot performs the dish washing task, a washing machine task and a garbage disposal task. A robot is able to navigate in the scene by using this spot information.

Right figures show 3D shape model of a washing machine, a dish and a kettle. handle information is displayed as red triangles and cylinders in the figures. The difference of the handle shape represents a difference of constraints. A red sphere in the kettle figure shows manipulation information.

\section{B. Tool manipulation motion planner using manipulation knowledge}

In order to generate whole body tool manipulation motions from trajectory of manipulate coordinate as an input data. Instead of constructing search space of dual arm joints and an object coordinate, which requires large search space., we propose a method by means of an object model with a manipulation knowledge and the whole body inverse kinematics technique,

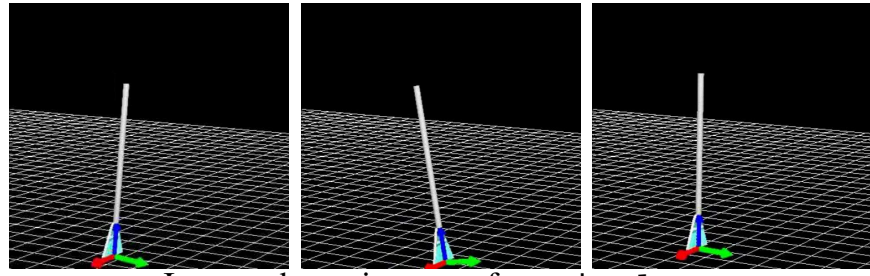

Input : the trajectory of manipulate

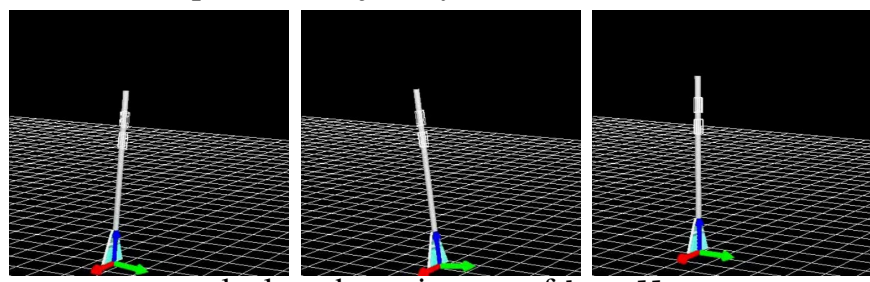

calculate the trajectory of handle

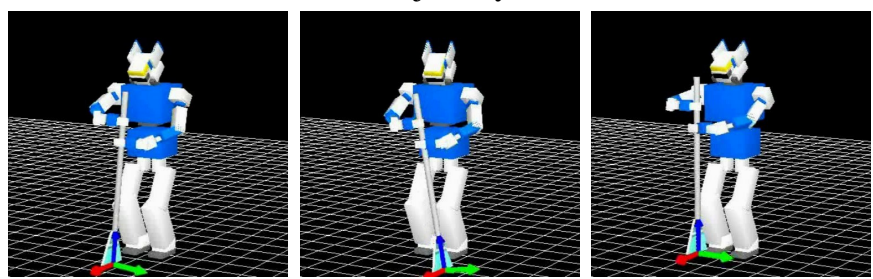

Output : the whole body posture sequence

Fig. 2. Procedure of the tool manipulation planner

this planner generates complex whole body motions (sequence of 30 D.O.F joint angles) from the simple input data (sequence of 6 D.O.F. coordinates).
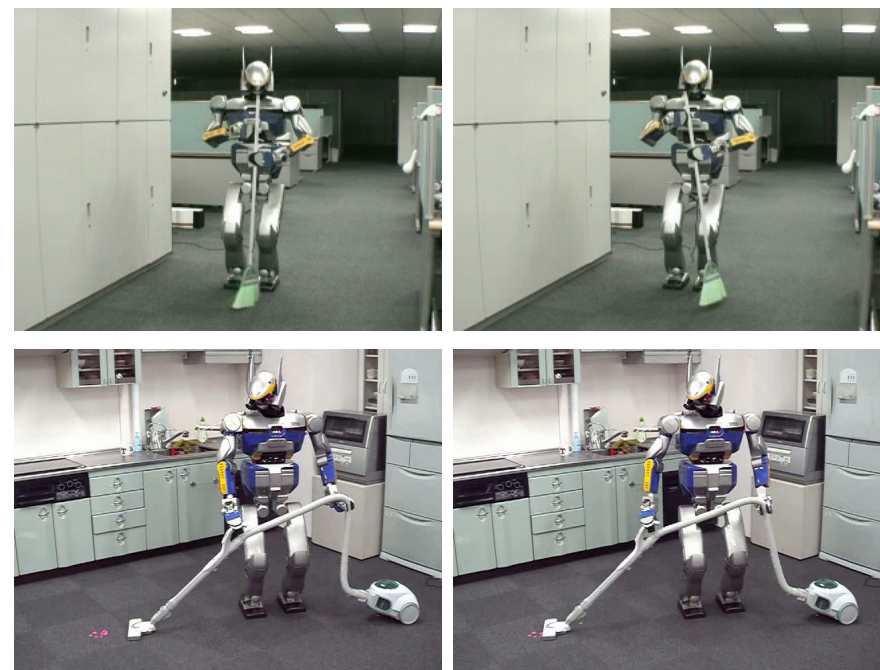

Fig. 3. Tool manipulation behaviors of a humanoid

Both sweeping and vacuuming behavior are able to generate using object models, tool manipulation knowledge and trajectories of a manipulation coordinate.

The procedure of generating tool manipulation motion is as followings

1) Input a trajectory of manipulate coordinates of a target object.

2) Calculate a trajectory of handle using relative coordinates between manipulate coordinate and handle coordinates.

3) Generate a whole body posture from each coordinates 

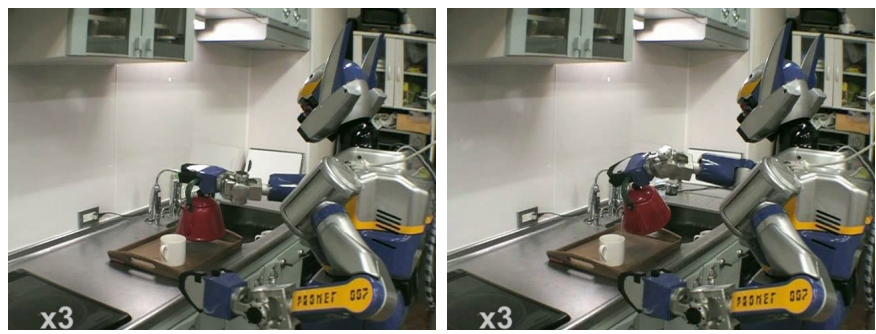

Fig. 4. Water pouring behavior of a life-sized humanoid robot by using tool manipulation motion planner.

and constraints of handle information by using the whole body IK method [13].

4) output whole body posture sequence by connecting each posture

Fig.2 shows the procedure of the tool manipulation planner. Upper figures shows the trajectory of the manipulate coordinate which is the input of this planner. Lower figures show the whole body posture sequence, which is the output of the planner. The tool manipulation planner described in this section generates complex whole body motions (sequence of 30 D.O.F. joint angles) from the simple input data (sequence of 6 D.O.F. coordinates).

Fig.3 shows tool manipulation behaviors of a life-sized humanoid robot by means of the tool manipulation planner. Both sweeping and vacuuming behavior are able to generate using object models with tool manipulation knowledge and trajectories of a manipulation coordinate.

\section{Motion generation programming in the water pouring example}

The motion of the water pouring behavior is programmed as followings by the use of tool manipulation motion planner.

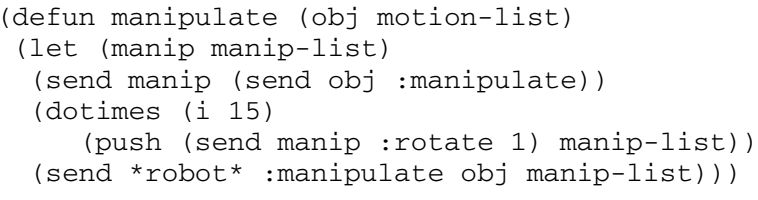

In the :manipulate method, it calls a :reach method which solves the whole body IK method to generate a whole body posture. Generated motions are shown in Fig.4.

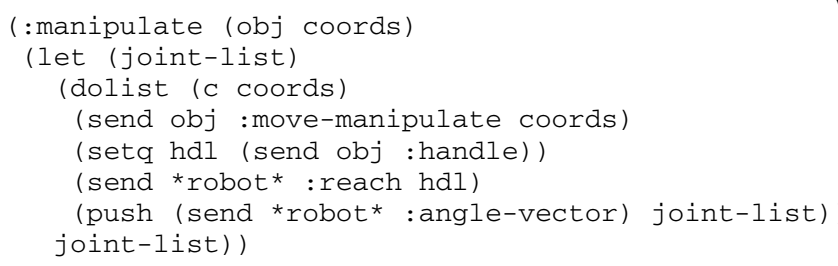

\section{BEHAVIOR VERIFICATION USING PLANNER BASED SENSORY NAVIGATION}

The idea of verification vision [14], [15] is to minimize uncertainty of an environment knowledge or to confirm the
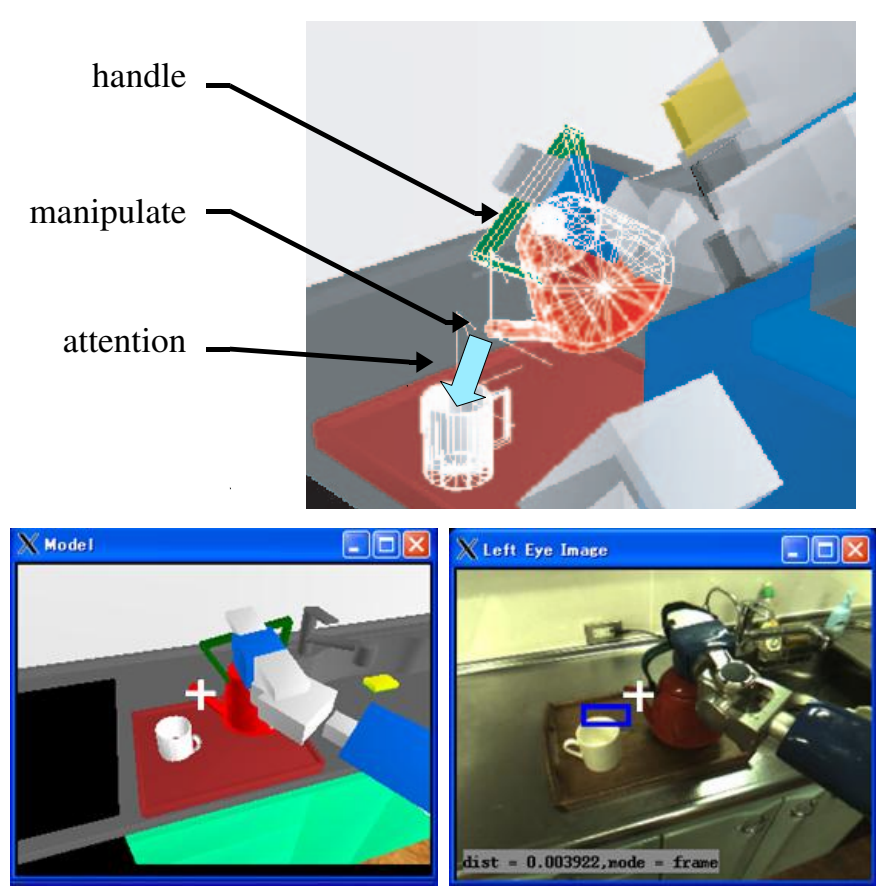

Fig. 5. Sensory navigation using an object model for behavior verification The robot grasp the kettle at handle position and rotate the kettle around the manipulate coordinates to pour water, while gazing at the attention area to verify then motion.

success of behavior by observing the environment before and after motion execution. The components of behavior verification are 1) which information is observed 2) which sensor processing carries necessary information. In the case of daily-life tasks, a robot must verify not only a location of target object, which is commonly used in assembly tasks, but also verify a change in an environment, in other words, event. For example, in the dishwashing task, the former is to confirm the position of a dish when picking or placing, the later is to confirm if a robot open and close a water outlet properly.

Previous researches of verification vision are highly taskspecific and assume the block world. Some researches provides systematic generation of sensing strategies [16], however they only consider assembly tasks that sensors only monitor locations and face contact relations of objects. In this paper, we propose an approach to generate verification motions by using motion planner environment that contains manipulation knowledge. Since this knowledge is associated with an object, our system can be considered as object-specific system, which handles event caused by manipulating an object.

The characteristics of behavior verification in this paper are 1) motion planner in our system is able to utilize both generating and verifying motion, since our system shares unique representation among vision, planning and controller 2) we developed a behavior management system to realize tight coupling of motion execution and sensory verification. 3 ) Our system does not limit to update locations of objects in the model but also detect event to control robot behavior. 


\section{A. An expansion of model description for behavior verification}

In order to perform behavior verification, we added following information to the manipulation knowledge described in II-A.

- Attention information: Coordinates on an object where a robot gaze and apply sensor processing function to verify motion.

An attention area on an image coordinates is able to calculate by using the $3 \mathrm{D}$ coordinates of an attention information, a 3D model of an object and a camera model (intrinsic parameter).

For example, in the upper image of Fig.5, the robot grasp the kettle at the handle position and rotate the kettle around the manipulate coordinates to pour water. In this situation, the robot gaze at the attention area to verify the motion, in order to confirm "does water comes or stops". The lower left figure shows view images and the attention area in motion planning engine and the lower right figure shows that of a camera captured view.

\section{B. Sensor information processing for behavior verification}

We have implemented following sensor information processing method for behavior verification: detect object using pattern matching technique (block matching, edge matching) or monitoring force information on the wrist, detect change in a view by using background difference, frame difference, or histogram difference.

\section{Visual verification programming in the water pouring ex- ample}

After several experiments, we found that the $S$ (Saturation value of HIS color space) histogram difference can detect the appearance and disappearance of water flow.

The similarity between two histogram is calculated by using Bhattacharyya [17] measure for the histogram. By using this equation, functions for detecting water flow are defined as followings and experimental results of applying these functions are shown inFig.6.

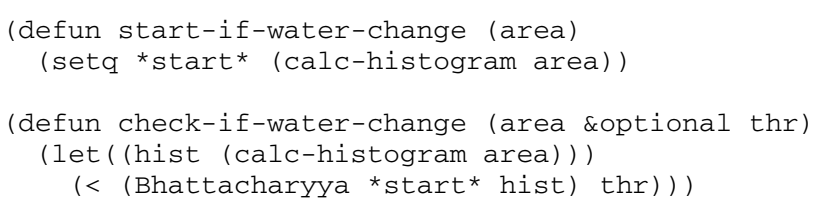

\section{Multi LAYERED BeHAVIOR MANAGEMENT} ARCHITECTURE

In order to realize concurrent motions that each part of a robot body move coordinately by single stream program, we have developed multi-layered behavior management architecture that consists of real-time layer, motion management layer, motion description layer.
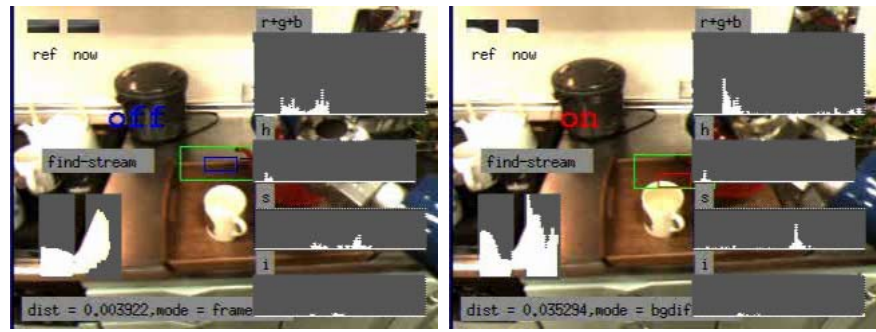

Fig. 6. An example of visual verification in water pouring task: The system detects water flow by calculating histogram difference.

\section{A. Motion modification primitive in real-time layer}

Real-time layer modify motions based on sensor information. Each feedback motion is called motion modification primitive. Motion modification primitives runs concurrently, to realize compliance control which is important when a robot interacts with external world.

Following three controller are main motion modification primitives, a stabilize controller for a lower body, a compliant controller for arms and a grasp controller for an end effecter. Theses modules run at $5[\mathrm{msec}]$ periodic loops.

\section{B. Motion primitive in motion management layer}

Motion primitives in the motion management layer control an execution of motion modification primitives. They assign parameters to modification primitives, start and examine processing termination.

We have implemented (1)walk that accepts a target location and generates walking motions for it, (2) reach that accepts a target coordinates and constraints in order to generate reaching motions, (3) grasp to control grasping and releasing.

\section{Realization of behavior verification with guarded motion}

A coordinate and constraints for the reach function corresponds to the handle information which is described in previous section. This motion primitive is implemented as a guarded motion manner, that the system executes motions while it satisfies sensory conditions. Therefore, variety of behavior evaluations are realized with the same reach function by alternating a condition function which corresponds to the sensor information processing for behavior verifications.

\section{Motion generation and visual verification programming in water pouring example}

The reach function is defined as followings:

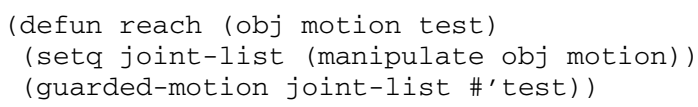

manipulate function is function defined in sectionII-C Guarded motion described above is defined as followings 

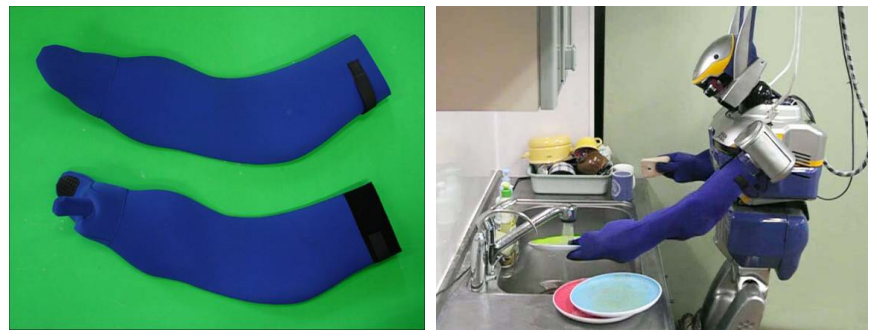

Fig. 7. Waterproof Glove for Humanoid

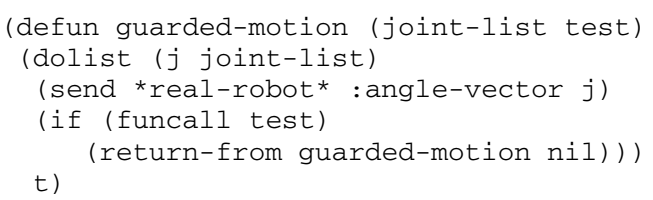

Finally, the program code to realize water pouring behavior from a kettle while verifying if the existence of water flow can be written as following.

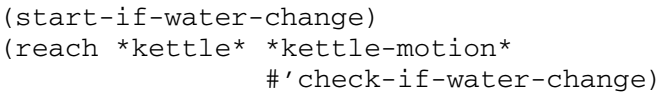

\section{BEHAVIOR VERIFICATION TASK EXPERIMENT USING HUMANOID ROBOT}

In order to demonstrate the effectiveness and importance of behavior verification function, we conducted dish washing behavior. We utilized the life-sized humanoid platform HRP-2 and attached waterproof glove.

\section{A. Waterproof Glove for Humanoid [18]}

Waterproof is one of the important function for a humanoid robot in the daily life environment where a robot have to perform tasks around water places such as a kitchen and a washing area. However, there ware a few researches on realizing a waterproof function. Tokyu Construction developed a rain coat like robot cloth and realized backhoe drive humanoid behavior in a rainy weather [19]. HRP-3P has a drip-proof joint module for outdoor applications [20].

In order to archive the waterproof function for humanoids in the daily life environment, totally waterproof is required.

We decided to select neoprene as material of a glove for a humanoid robot. Fig.7 shows the developed waterproof glove and a dish washing experiment.

\section{B. Visual navigation and processing for behavior verification}

When the robot turns on the water outlet to run water, it verify if the water runs or not by using visual information. The upper left image in the Fig.8 shows simulated view information when the system generates the behavior with 3D model of an environment and manipulation knowledge. The upper right image shows camera view captured by the robot along with super imposed visible edges of the 3D model. Lower images show attention area by projecting attention
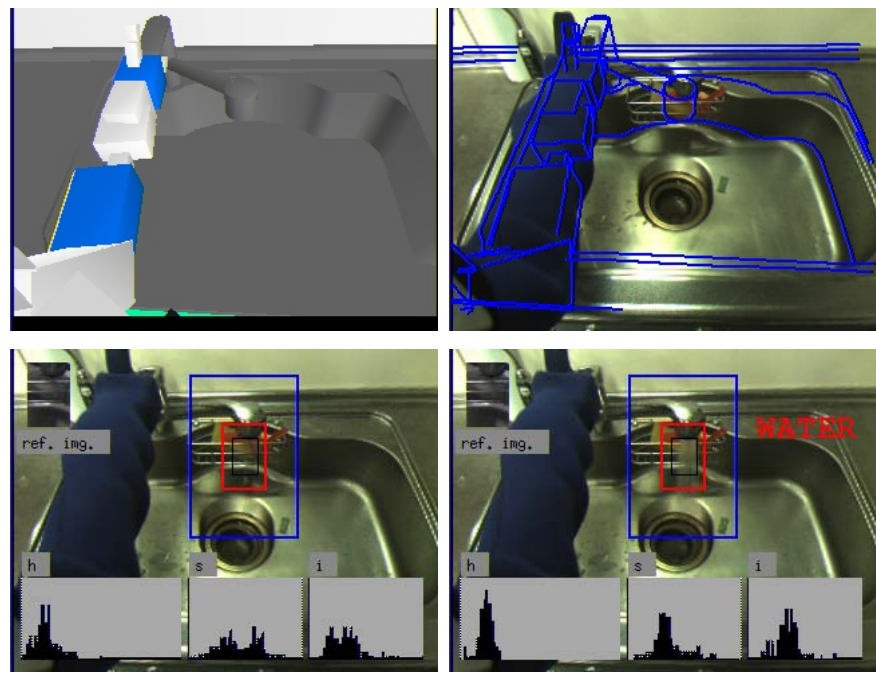

Fig. 8. Visual navigation and processing for behavior verification The upper left image shows simulated view information and camera view. Lower images show attention area by projecting attention coordinates to the camera image plane and the result water flow detection using HSI histogram

coordinates to the camera image plane. The red rectangle shows attention area and the area is keep tracked to respond to view changes caused by stabilizing motion. In order to detect water flow, we use HSI color histogram in the red rectangle. The existence of water flow is determined by the difference of index number of the histogram peek of S(saturation value) between current and background pixels in the area.

\section{Motion generation and behavior verification in wash dish experiment}

Fig.9 shows motion generation and behavior verification in washing dish experiment. (1), (3), (5), (6) shows motion generation phase using planner. (2) shows the robot examines if there exists water flows by navigating visual attention around the water outlet. In (4), the robot gaze at a human face where the system predict.

\section{CONCLUSION}

This paper describes an on-going project to realize a dailylife helping humanoid. Tool manipulation behavior is essentially important for this aim, thus we selected the model based approach with 3D shape information and manipulation knowledge. This paper presents behavior verification to overcome the drawback of this approach. The contributions of this paper are summarized as followings:

1) We propose behavior verification system based on manipulation motion planner system toward automatic generation of sensory tasks.

2) Practical manipulate motion planner which has simple interface to generate whole body posture sequence are presented.

3) We developed a visual processing method to detect a water flow by using the histogram comparison technique.

4) We designed and implemented multilayered behavior management architecture that provides single stream 


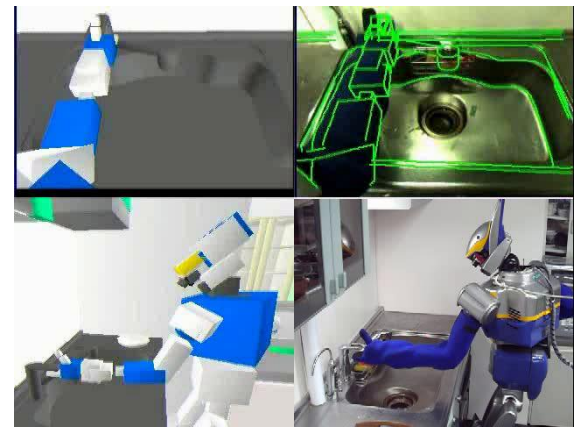

(1)pushing tap (plan)

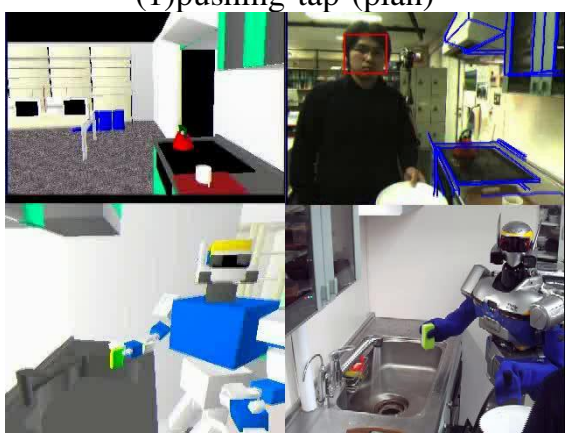

(4)detect human(verify)

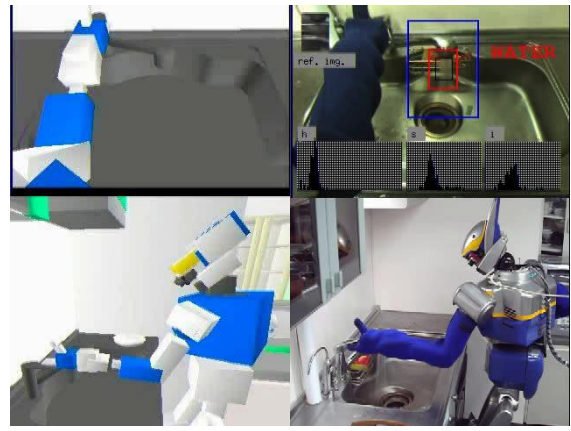

(2)detect water flow(verify)

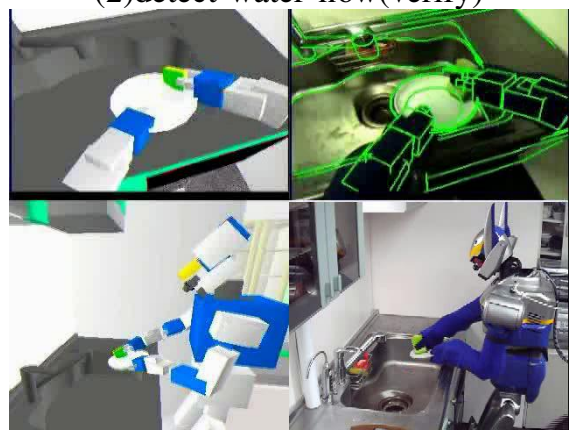

(5)washing behavior(plan)

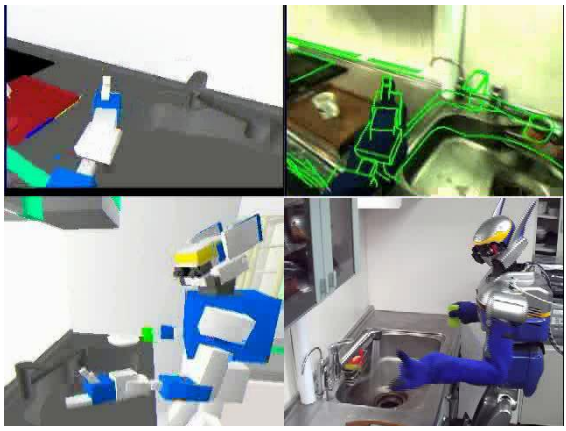

(3)gazing to human(plan)

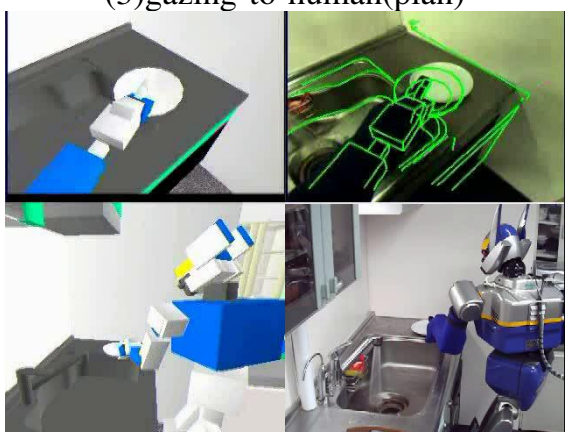

(6)putting a dish(plan))

Fig. 9. Motion generation and behavior verification in wash dish experiment

programming model to users for realizing concurrent motions

5) We demonstrated water pouring task and washing dish task in real environment while verifying its own motion.

\section{REFERENCES}

[1] R.A. Brooks and L.A.Stein. Building Brain for Bodies. In Autonomous Robots, Vol. 1, pp. 7-25, 1994

[2] Y. Sakagami, R. Watanabe, C. Aoyama, S. Matsunaga, N. Higaki, and K. Fujimura. The intelligent ASIMO: System overview and integration. In Proceedings of the 2002 IEEE/RSJ International Conference on Intelligent Robots and Systems (IROS'02), pp. 2478-2483, 2002.

[3] K. Okada, M. Inaba, and H. Inoue. Integration of real-time binocular stereo vision and whole body information for dynamic walking navigation of humanoid robot. In Proceedings of International Conference on Multisensor Fusion and Integration for Intelligent Systems (MFI'03), pp. 131-136, 2003.

[4] J.-S. Gutmann, M. Fukuchi, and M. Fujita. A floor and obstacle height map for 3d navigation of a humanoid robot. In Proceedings of the 2005 IEEE International Conference on Robotics and Automation (ICRA '05), 2005.

[5] J.F. Seara, K.H. Strobl, and G. Schmidt. Path-dependent gaze control for obstacle avoidance in visual guided humanoid walking. In Proceedings of the IEEE/RSJ International Conference on Intelligent Robots and Systems (IROS'03), pp. 887-892, 2003.

[6] R. Dillmann, P. Steinhaus, and R. Becher. ARMAR II - A Learning and Cooperative Multimodal Humanoid Robot. International Journal on Humanoid Robotics, Vol. 1, No. 1, pp. 143-156, 2004.

[7] R. Ambrose and S. Askew and W. Bluethmann and M. Diftler Humanoids Designed to do Work. In In Proceedings of the IEEE International Conference on Humanoid Robots (Humanoids 2001), pp. 173-180, 2001.

[8] N. Sien and T. Sakaguchi and K. Yokoi and Y. Kawai and K. Maruyama Operating Humanoid Robots in Human Environments. In RSS 2006 Workshop: Manipulation for Human Environments, 2006.

[9] K. Okada, T. Ogura, A. Haneda, J. Fujimoto, F. Gravot, and M. Inaba Humanoid Motion Generation System on HRP2-JSK for Daily Life Environment. In Proc. of International Conference on Mechatronics and Automation (ICMA'05), pp. 1772-1777, 2005.
[10] J.J. Kuffner and K. Nishiwaki and S. Kagami and M. Inaba and H. Inoue. Motion planning for humanoid robots. In In Proceedings of 11th International Symposyum on Robotics Research (ISRR'03), p. 20, 2003.

[11] O. Lorch, A. Albert, J. Denk, M. Gerecke, R. Cupec, J.F. Seara, W. Gerth, and G. Schmidt. Experiments in vision-guided biped walking. In Proceedings of the IEEE/RSJ International Conference on Intelligent Robots and Systems (IROS'02), pp. 2484-2490, 2002.

[12] F. Gravot, R. Alami, and T. Simeon. Playing with several roadmaps to solve manipulation problems. In Proceedings of the 2002 IEEE International Conference on Robotics and Automation, pp. 2311-2316, 2002.

[13] K. Yamane and Y. Nakamura. Synergetic CG Choreography through Constraining and Deconstraining at Will. In Proceedings of the 2002 IEEE International Conference on Robotics and Automation (ICRA '02), pp. 855-862, 2002.

[14] R.C.Bolles. Verification Vision for Programmable Assembly. In Proceedings of 5th International joint Conference on Artificial Intelligence, pp. 579-575, 1977.

[15] R.D. Rimey. Control of Selective Perception using Bayes Nets and Decision Theory. Technical Report TR468, The University of Rochester, 1993

[16] J. Miura and K. Ikeuchi. Task-Oriented Generation of Visual Sensing Strategies in Assembly Tasks. IEEE Transactions on Pattern Analysis and Machine Intelligence, Vol. 20, No. 2, pp. 126-138, 1998.

[17] T. Kailath. The Divergence and Bhattacharyya Distance Measures in Signal Selection. IEEE Transaction on Communication Technology, Vol. 15, pp. 52-60, 1967.

[18] K. Okada, M. Hayashi, and M. Inaba. Development of Waterproof Glove for Humanoid Robots in Daily Environment Tasks. In Proc. of International Conference on Robotics and Automation (ICRA'06), pp. 4419-4421, 2006

[19] Tokyu Construction, Patent Publication Number 2004-183157, Japan. Protection wear For Humanoid Robot.

[20] T. Isozumi, K. Akachi, N. Kanehira, K. Kaneko, F. Kanehiro, an H. Hirukawa. The development of Humanoid Robot HRP-2P. In Proceedings of the 21th Annual Conference of Robotics Society of Japan, p. 3L11, 2004 (in Japanese). 